\title{
On Choosing Between Deterministic and Indeterministic Models: Underdetermination and Indirect Evidence
}

\author{
Forthcoming in Synthese
}

Charlotte Werndl, Lecturer, c.s.werndl@lse.ac.uk

\author{
Department of Philosophy, Logic and Scientific Method \\ London School of Economics
}

\begin{abstract}
There are results which show that measure-theoretic deterministic models and stochastic models are observationally equivalent. Thus there is a choice between a deterministic and an indeterministic model and the question arises: Which model is preferable relative to evidence? If the evidence equally supports both models, there is underdetermination. This paper first distinguishes between different kinds of choice and clarifies the possible resulting types of underdetermination. Then a new answer is presented: the focus is on the choice between a Newtonian deterministic model supported by indirect evidence from other Newtonian models which invoke similar additional assumptions about the physical systems and a stochastic model that is not supported by indirect evidence. It is argued that the deterministic model is preferable. The argument against underdetermination is then generalised to a broader class of cases. Finally, the paper criticises the extant philosophical answers in relation to the preferable model. Winnie's (1998) argument for the deterministic model is shown to deliver the correct conclusion relative to observations which are possible in principle and where there are no limits, in principle, on observational accuracy (the type of choice Winnie was concerned with). However, in practice the argument fails. A further point made is that Hoefer's (2008) argument for the deterministic model is untenable.
\end{abstract}




\section{Contents}

1 Introduction 3

2 Deterministic and Indeterministic Models 4

2.1 Deterministic Models . . . . . . . . . . . . . . . . 4

2.2 Stochastic Models . . . . . . . . . . . . . . . . 7

3 Observational Equivalence of Deterministic and Indeterministic Models

4 Choice and Underdetermination between Deterministic and Indeterministic Models

5 A New Argument Against Underdetermination Based on Indirect Evidence

6 Discussion of the Previous Philosophical Literature 20

6.1 Winnie's Argument for the Deterministic Model . . . . . . . . . 20

6.2 Hoefer's Argument for the Deterministic Model . . . . . . . . . 23

$\begin{array}{lll}7 & \text { Conclusion } & 25\end{array}$

$\begin{array}{lr}\text { References } & 26\end{array}$ 


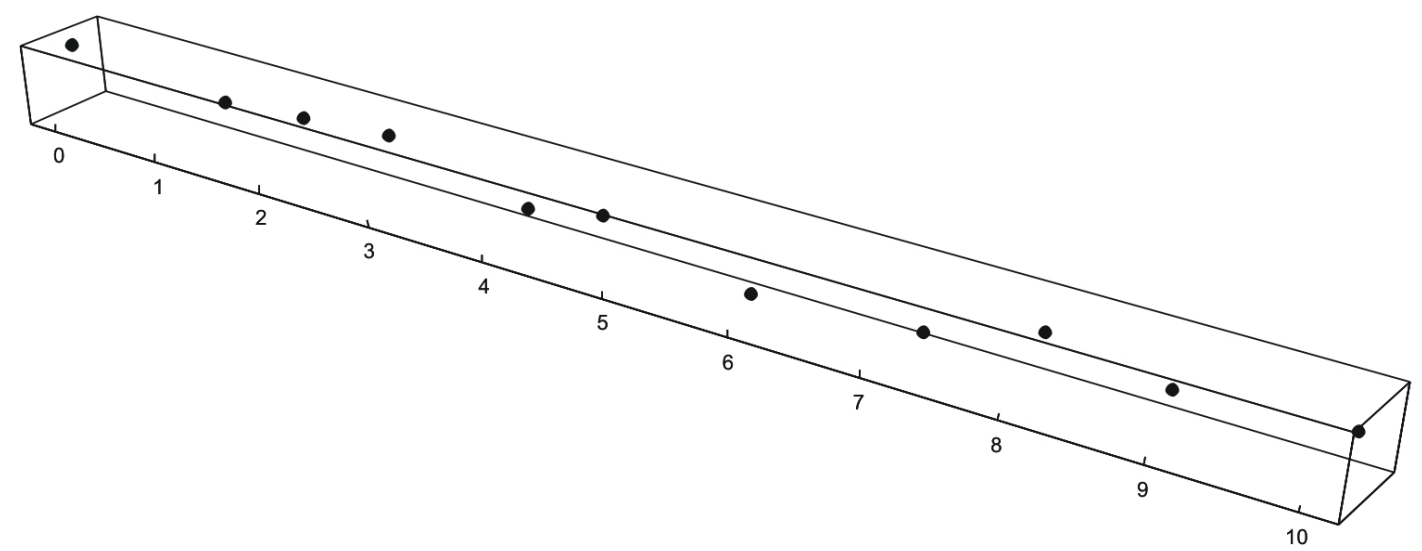

Figure 1: Observed position of a particle at time $0,1, \ldots 9,10$ (observed positions are values in the unit square.

\section{Introduction}

Consider the evolution of the position of a particle. An observer of this physical process obtains a sequence of observations such as the one shown in Figure 1. This sequence represents the observed position of the particle at time $0,1, \ldots$ 9, 10 (observed positions are values in the unit square). Scientists aim to find a model which reproduces these observations and which gives correct predictions. A question that arises is whether the physical system is best described by a deterministic or an indeterministic model and opinion might be that the observations only allow for one or the other. However, recent results on observational equivalence show that in several cases, including the example of the position of the particle, both a deterministic and an indeterministic model can account for the observations. If the evidence equally supports both models, there is underdetermination. Thus, the central question of this paper is: Is a deterministic or an indeterministic model preferable relative to evidence?

To provide a basis for answering this question, Section 2 introduces the relevant deterministic and indeterministic models and Section 3 presents the results on observational equivalence between deterministic and indeterministic models. Section 4 then distinguishes between different kinds of choice and clarifies the possible resulting types of underdetermination. Section 5 presents a novel answer to the central question: the focus is on the choice between a Newtonian deterministic model supported by indirect evidence from other Newtonian models which invoke confirmed similar additional assumptions about the physical systems and a stochastic model that is not supported by indirect evidence. The examples of choice between deterministic and stochastic models discussed in the extant philosophical literature fall under this class.

The argument presented is that the deterministic model is preferable. The argument against underdetermination is then generalised to a much broader 
class of cases. Section 6 subsequently discusses the extant philosophical answers in relation to the preferable model. Winnie's (1998) argument for the deterministic model is shown to deliver the correct conclusion relative to observations which are possible in principle and where there are no limits, in principle, on observational accuracy (the type of choice Winnie was concerned with). However, for the other kinds of choice, in particular the ones arising in practice, the argument fails. A further point made is that Hoefer's (2008) argument for the deterministic model is untenable. Finally, Section 7 summarises the results.

\section{Deterministic and Indeterministic Models}

First of all, it should be mentioned that the term 'model' as understood in this paper refers to a set of solutions (and not, as in the semantic view, to a full solution). ${ }^{1}$ The deterministic and indeterministic models of concern in this paper are measure-theoretic deterministic models and stochastic models. There are two kinds of models: discrete-time models, wherein time increases in discrete steps, and continuous-time models involving a continuous time-parameter. As relevant results are easier to understand for discrete time, this paper focuses on discrete-time models. All results, though, can be applied to continuous-time models.

\subsection{Deterministic Models}

This paper is concerned with measure-theoretic deterministic models, i.e., deterministic models denoted by $\left(M, T_{t}, p\right)$. Here $M$ is a set, called the phase space, where each $m \in M$ represents a possible state of the deterministic system (i.e., $m$ is a variable representing the state of the system). $T_{t}: M \rightarrow M$, where $t$ ranges over $\mathbb{Z}$, are bijective maps with $T_{t+s}=T_{t}\left(T_{s}\right)$ for all $t, s$; the family of maps $T_{t}$ describes the dynamics of the system, namely it describes that each state $m \in M$ evolves to $T_{t}(m)$ after $t$ time steps. And $p$ is a probability measure which assigns a probability to regions of $M .^{2}$ Clearly, models thus defined are deterministic according to the standard philosophical definition, namely that a model is deterministic iff any two solutions that agree at one time agree at all times (Butterfield 2005).

The solution through $m$ represents a possible path of the deterministic system over time. Formally, it is the bi-infinite sequence $\left(\ldots, T_{-1}(m), T_{0}(m), T_{1}(m), \ldots\right)$; $T_{t}(m)$ is called the $t$-th iterate of $m$. Deterministic models where the probability is invariant under time-evolution, i.e., where $p\left(T_{t}(A)\right)=p(A)$ for all regions $A$

\footnotetext{
${ }^{1}$ So models are not individuated as under the semantic view of theories.

${ }^{2}$ There are various interpretations of this probability measure. For example, according to the time-average interpretation, the probability of $A$ is the long-run average of the proportion of time a solution spends in $A$ (cf. Lavis 2011).
} 


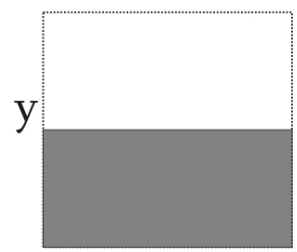

$\mathbf{X}$

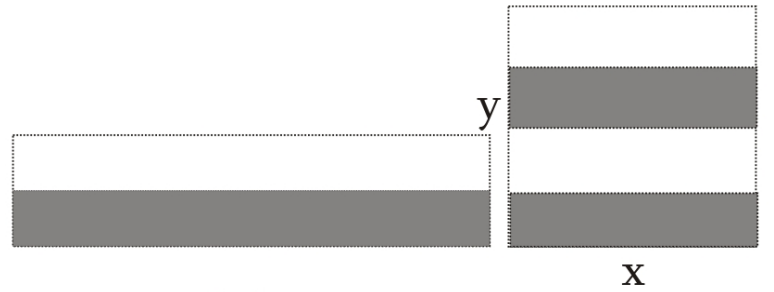

(a)

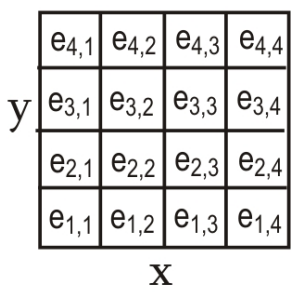

(b)

Figure 2: (a) the baker's map $T((x, y))$; (b) the observation function $\Phi_{16}$

of $M$ and all $t$ are called measure-preserving deterministic models. Deterministic models, in particular measure-preserving deterministic models, are among the most important deterministic models in science. For instance, all deterministic models in Newtonian theory that model energy-conserving systems are measure-preserving (Petersen 1989, 5-6).

In the observation of a deterministic system, a value of the variable representing the state of the system is observed that is dependent on, but typically different from, the actual value of the variable. This is because observation cannot be done with infinite precision (and so observation corresponds to coarsegraining the phase space). Mathematically, an observation can be modelled by an observation function, i.e., a function $\Phi: M \rightarrow M_{O}$, where $\Phi(m)$ represents the observed value and $M_{O}$ is the set of all possible observed values. Finitevalued observation functions are observation functions with a finite number of values. A finite-valued observation function $\Phi$ is nontrivial iff more than one value can be seen, i.e., there is a value $o$ with $0<p(\{m \in M \mid \Phi(m)=o\})<1$. An observation function $\Xi$ is at least as fine as an observation function $\Phi$ iff for any value $o$ of $\Xi$ there is a value $q$ of $\Phi$ such that for all $m \in M$ if $\Xi(m)=o$, then $\Phi(m)=q$. An observation function $\Xi$ is finer than an observation function $\Phi$ iff it is at least as fine as $\Phi$ and there are values $o_{1}, o_{2}$ of $\Xi$ and a value $q$ of $\Phi$ such that for all $m \in M$ if $\Xi(m)=o_{1}$ or $\Xi(m)=o_{2}$, then $\Phi(m)=q$ (i.e., there are two values of $\Xi$ to which $\Phi$ assigns the same value). Observation functions can represent actual or hypothetical observations.

The following two examples illustrate deterministic models.

Example 1: The baker's model.

Consider the physical system of a particle which starts its motion from a square and moves in the direction perpendicular to the square (assuming there is no friction). The particle bounces on several mirrors, causing it to return to the square where the process starts again. Mirrors are of three kinds: pairs of mirrors of parabolic shape that lead to divergence or convergence of paths; pairs of straight mirrors that shift the paths to the left, right, up or down; mirrors at corners that direct the particle back to the square (see Pitowsky 1995, 166). 


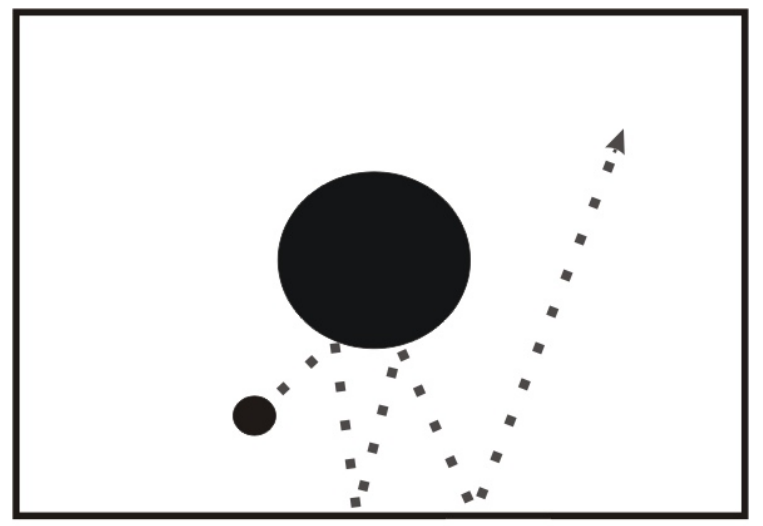

Figure 3: A billiard system with a convex obstacle

This physical system can be described by a Newtonian deterministic model $\left(M, T_{t}, p\right)$ called the baker's model. Here $M$ is the unit square $[0,1] \times[0,1]$, and $p$ is the uniform probability measure on the unit square. A particle in initial state $(x, y)$ bounces on the mirrors and returns to the square at $T((x, y))$ where

$$
T((x, y)):=\left(2 x, \frac{y}{2}\right) \text { if } 0 \leq x<\frac{1}{2} ;\left(2 x-1, \frac{y+1}{2}\right) \text { if } \frac{1}{2} \leq x \leq 1 .
$$

Finally, $T_{t}((x, y)):=T^{t}((x, y))$ ( $T$ applied $t$ times). Figure 2(a) shows that $T$ first stretches the unit square to twice its length and shrinks it to half its width; then, the rectangle obtained is cut in half and the right half is stacked on top of the left. (Parabolic mirrors lead to the multiplication of the value of a coordinate by a constant, and straight mirrors lead to the addition of a constant to the value of a coordinate, see Pitowsky 1995, 166).

Consider the observation function $\Phi_{16}$ of the baker's map with sixteen values $e_{i, j}=\left(\frac{2 i+1}{8}, \frac{2 j+1}{8}\right), 0 \leq i, j \leq 3$, assigned as follows: $\Phi_{16}((x, y))=\left(\frac{2 k+1}{8}, \frac{2 l+1}{8}\right)$ for $\frac{k}{4} \leq x<\frac{k+1}{4}$ and $\frac{l}{4} \leq y<\frac{l+1}{4}, k, l \in \mathbb{N}, 0 \leq k, l \leq 3$. Figure 2(b) shows this observation function; i.e., for all states $m$ in the bottom left box the value $e_{1,1}$ is observed, etc. Suppose that the baker's model is initially in state $(0.824,0.4125)$. Then the first 11 iterates coarse-grained by the observation function $\Phi_{16}$ are: $\left(\Phi_{16}\left(T_{0}((0.824,0.4125))\right), \Phi_{16}\left(T_{1}((0.824,0.4125))\right), \ldots, \Phi_{16}\left(T_{10}((0.824,0.4125))\right)\right)=$ $\left(\left(\frac{7}{8}, \frac{3}{8}\right),\left(\frac{5}{8}, \frac{5}{8}\right),\left(\frac{3}{8}, \frac{7}{8}\right),\left(\frac{5}{8}, \frac{3}{8}\right),\left(\frac{1}{8}, \frac{5}{8}\right),\left(\frac{3}{8}, \frac{3}{8}\right),\left(\frac{5}{8}, \frac{1}{8}\right),\left(\frac{3}{8}, \frac{5}{8}\right),\left(\frac{7}{8}, \frac{3}{8}\right),\left(\frac{7}{8}, \frac{5}{8}\right),\left(\frac{7}{8}, \frac{7}{8}\right)\right)$. This is the sequence shown in Figure 1. Consequently, an observation of the baker's system can yield the time series shown in Figure 1.

Example 2. Newtonian models of billiards with convex obstacles.

Consider the physical system of a billiard ball which moves on a rectangular table (assuming there is no friction). The billiard table differs from a normal one because there are a finite number of convex obstacles on the table. Suppose the evolution of this physical system is modelled at discrete-time intervals $t r_{0}$, where 
$r_{0} \in \mathbb{R}^{+}$is an arbitrary constant, $t \in \mathbb{Z}$. Figure 3 shows such a billiard system (grey dots indicate the position of the ball at discrete-time intervals). Such a billiard system can be described by a deterministic Newtonian model $\left(M, T_{t}, p\right)$ (cf. Orstein and Galavotti 1974). Here $M$ represents the set of all possible states. It is the set of all possible vectors consisting of the value representing the position and the value representing the direction of the velocity of the ball (all that matters for the construction of phase space is the direction of the velocity since speed is constant). $T_{t}(m)$ gives the position and direction after $t r_{0}$ time units of the ball that starts out in initial position and initial direction $m . p$ is the normalised Lebesgue measure and assigns the probability $p(A)$ to the event that the billiard system takes one of the states represented by $A$ for any arbitrary region $A$ of phase space. The solution through $m$ is the sequence $\left(\ldots, T_{-1}(m), T_{0}(m), T_{1}(m), \ldots\right)$, representing the path of the billiard system which is initially in $m$ (over the time points $t r_{0}, t \in \mathbb{Z}$ ).

\subsection{Stochastic Models}

The indeterministic models of concern in this paper are stochastic models, which represent physical systems that evolve according to probabilistic laws. A stochastic model is denoted by $\left\{Z_{t}\right\}$ and consists of a family of functions $Z_{t}: \Omega \rightarrow E$ with $t \in \mathbb{Z}$, where the functions $Z_{t}$ also specify the probability distributions of the model. $E$ represents the set of all possible outcomes, called the outcome space. Each $\omega \in \Omega$ encodes a possible path of the stochastic system in all its detail and is usually unknown in practice (hence $\Omega$ is the set encoding all possible paths), and $Z_{t}(\omega)$ represents the outcome of the system at time $t$. The probability distributions characterise the probabilistic behaviour: e.g., the probability distribution $P\left(Z_{t} \in A\right)$ gives one the probability that the outcome of the system is in $A$ at time $t$ for any region $A$ of $E$ and $t \in \mathbb{Z}$. Joint probability distributions tell one the probabilities of outcomes at different times, such as $P\left(Z_{s} \in A\right.$ and $\left.Z_{t} \in B\right)$; and conditional probability distributions $P\left(Z_{s} \in A\right.$ given that $\left.Z_{t} \in B\right)$ tell one the probability that the outcome is in $A$ at time $s$ given that it is in $B$ at time $t$ (for any $t, s$ and any arbitrary regions $A, B$ of $E)$.

A realisation represents a possible path of the stochastic system over time. It is a bi-infinite sequence $\left(\ldots, Z_{-1}(\omega), Z_{0}(\omega), Z_{1}(\omega), \ldots\right)$ for an arbitrary $\omega \in \Omega$. For stochastic models there is usually indeterminism - given the initial outcome, there are several possible outcomes that might follow, and these possibilities are measured by probabilities. Most indeterministic models used are stochastic models, which are ubiquitous in science.

Observations of stochastic systems are also modelled by observation functions, i.e., functions $\Gamma: E \rightarrow E_{O}$, where $\Gamma(e)$ represents the observed value and $E_{O}$ is the set of all possible observed values. Finite-valued observation functions 
are observation functions with a finite number of values. That an observation function $\Delta$ is at least as fine as an observation function $\Gamma$ and that an observation function $\Delta$ is finer than an observation function $\Gamma$ is exactly defined as for observation functions of deterministic models (cf. Subsection 2.1).

The following models are among the most widely-known stochastic models:

Example 3: Markov models.

In Markov models the next outcome depends only on the previous outcome. The formal definition of a Markov model $\left\{Z_{t}\right\}$ proceeds as follows: (i) the outcome space consists of $N$ states $E=\left\{e_{1}, \ldots, e_{N}\right\}, N \in \mathbb{N}$; (ii) and the next outcome only depends on the previous one, i.e., $P\left(Z_{t}=e_{h}\right.$ given $\left.Z_{t-1}, Z_{t-2} \ldots, Z_{j}\right)=$ $P\left(Z_{t}=e_{h}\right.$ given $\left.Z_{t-1}\right)$ for all $t \in \mathbb{Z}$, all $j \leq t-1$ and all outcomes $e_{h}$.

Let us consider a specific Markov model $\left\{W_{t}\right\}$ defined as follows. There are sixteen possible states $e_{i, j}:=\left(\frac{2 i+1}{8}, \frac{2 j+1}{8}\right)$, and $P\left(e_{i, j}\right)=1 / 16,0 \leq i, j \leq 3$. Each present state can be followed by two other states, and the probability is $1 / 2$ that the present state is followed by any of these two states. More specifically, $\left\{W_{t}\right\}$ is defined such that state $e_{1,1}$ is followed by state $e_{1,1}$ or state $e_{1,2}, e_{1,2}$ by $e_{1,3}$ or $e_{1,4}, e_{1,3}$ by $e_{3,1}$ or $e_{3,2}, e_{1,4}$ by $e_{3,3}$ or $e_{3,4}, e_{2,1}$ by $e_{1,1}$ or $e_{1,2}, e_{2,2}$ by $e_{1,3}$ or $e_{1,4}, e_{2,3}$ by $e_{3,1}$ or $e_{3,2}, e_{2,4}$ by $e_{3,3}$ or $e_{3,4}, e_{3,1}$ by $e_{2,1}$ or $e_{2,2}, e_{3,2}$ by $e_{2,3}$ or $e_{2,4}, e_{3,3}$ by $e_{4,1}$ or $e_{4,2}, e_{3,4}$ by $e_{4,3}$ or $e_{4,4}, e_{4,1}$ by $e_{2,1}$ or $e_{2,2}, e_{4,2}$ by $e_{2,3}$ or $e_{2,4}, e_{4,3}$ by $e_{4,1}$ or $e_{4,2}$, $e_{4,4}$ by $e_{4,3}$ or $e_{4,4}$. For one of the realisations of $\left\{W_{t}\right\}$ the entries from time 0 to 10 are: $\left(e_{2,4}, e_{3,3}, e_{4,2}, e_{2,3}, e_{3,1}, e_{2,2}, e_{1,3}, e_{3,2}, e_{2,4}, e_{3,4}, e_{4,4}\right)$. This is the sequence shown in Figure 1. Therefore, the time series of Figure 1 can arise from a Markov model. Recall that this time series can also arise from the baker's model. These statements hint at the results concerning observational equivalence between deterministic and stochastic models. What follows in Section 3 is a discussion of these results.

\section{Observational Equivalence of Deterministic and Indeterministic Models}

What is meant by observational equivalence is that the deterministic model, relative to an observation function $\Phi$, and the stochastic model, relative to an observation function $\Gamma$, give the same predictions. Therefore, the notion of observational equivalence is a relation between a deterministic model, an observation function of a deterministic model, a stochastic model and an observation function of a stochastic model. The phrase 'give the same predictions' needs further elaboration. Relative to an observation function, the predictions obtained from a stochastic model are the probability distributions over its realisations coarse-grained by the observation function. A deterministic model comes equipped with a probability measure $p$. Hence, relative to an observa- 
tion function, the predictions derived are the probability distributions over the solutions coarse-grained by the observation function. Therefore, a stochastic model $\left\{Z_{t}\right\}$ to which an observation function $\Gamma$ is applied and a deterministic model $\left(M, T_{t}, p\right)$ to which an observation function $\Phi$ is applied give the same predictions iff: (i) possible values of $\Gamma$ and of $\Phi$ are the same, and (ii) probability distributions over the realisations of the stochastic model coarse-grained by $\Gamma$ are the same as probability distributions over the solutions of the deterministic model coarse-grained by $\Phi$.

Suppose that a deterministic model $\left(M, T_{t}, p\right)$ and an observation function $\Phi: M \rightarrow M_{O}$ are given. Can a stochastic model observationally equivalent to $\left(M, T_{t}, p\right)$ be found? The answer is affirmative: $\left\{Z_{t}\right\}:=\left\{\Phi\left(T_{t}\right)\right\}$ is a stochastic model. It is constructed by applying the observation function to the deterministic model. Assuming that the observation function of the stochastic model is the identity function (i.e., all outcomes of the stochastic system can be observed), the possible observed values of $\left\{\Phi\left(T_{t}\right)\right\}$ are the possible observed values of $\left(M, T_{t}, p\right)$; and the realisations of $\left\{\Phi\left(T_{t}\right)\right\}$ and the solutions of $\left(M, T_{t}, p\right)$ coarse-grained by $\Phi$ have the same probability distributions. Therefore, the stochastic model $\left\{\Phi\left(T_{t}\right)\right\}$, relative to the identity function, and $\left(M, T_{t}, p\right)$, relative to $\Phi$, are observationally equivalent. This result can easily be generalised to the case where the observation function of the stochastic model is not the identity function. Clearly, for any arbitrary functions $\Psi$ and $\Gamma$ with $\Gamma(\Psi)=\Phi$, we have $\left\{\Phi\left(T_{t}\right)\right\}=\left\{\Gamma\left(\Psi\left(T_{t}\right)\right)\right\}$. Therefore, $\left(M, T_{t}, p\right)$ relative to the observation function $\Phi$ is observationally equivalent to the stochastic model $\left\{\Psi\left(T_{t}\right)\right\}$ relative to the observation function $\Gamma$. Hence, at this observation level, the physical system can be modelled by the deterministic model $\left(M, T_{t}, p\right)$ or the stochastic model $\left\{\Psi\left(T_{t}\right)\right\}$.

This result can be illustrated with the example of the physical system of a particle bouncing on mirrors. It is known that this physical model can be described by a deterministic Newtonian model, namely the baker's model (Example 1). Now consider the observation function $\Phi_{16}$ (see Figure 4 ). The set of possible values of $\Phi_{16},\left\{e_{i, j} \mid 0 \leq i, j \leq 3\right\}$, is also the set of all possible outcomes of the stochastic model $\left\{Z_{t}\right\}=\left\{\Phi_{16}\left(T_{t}\right)\right\}$; and the probability distributions of this stochastic model are determined by applying $\Phi_{16}$ to the baker's model. As shown in Figure 4, examples for these identical probability distributions are: $p\left(\Phi\left(T_{t}\right)=e_{1,1}\right)=P\left(Z_{t}=e_{1,1}\right)$ or $p\left(\Phi\left(T_{t+1}\right)=e_{4,4}\right.$ given that $\left.\Phi\left(T_{t}\right)=e_{3,4}\right)=P\left(Z_{t+1}=e_{4,4}\right.$ given that $\left.Z_{t}=e_{3,4}\right)$ for all $t \in \mathbb{Z}$. It follows that the deterministic baker's model, relative to $\Phi_{16}$, and the stochastic model $\left\{\Phi_{16}\left(T_{t}\right)\right\}$, relative to the identity function (i.e., all the outcomes of the stochastic system can be observed), are observationally equivalent. ${ }^{3}$ Thus, at

\footnotetext{
${ }^{3}$ The physical systems of a particle bouncing on mirrors and of billiards with convex obstacles involve strong idealising assumptions - in particular, that there is no friction. Conse-
} 


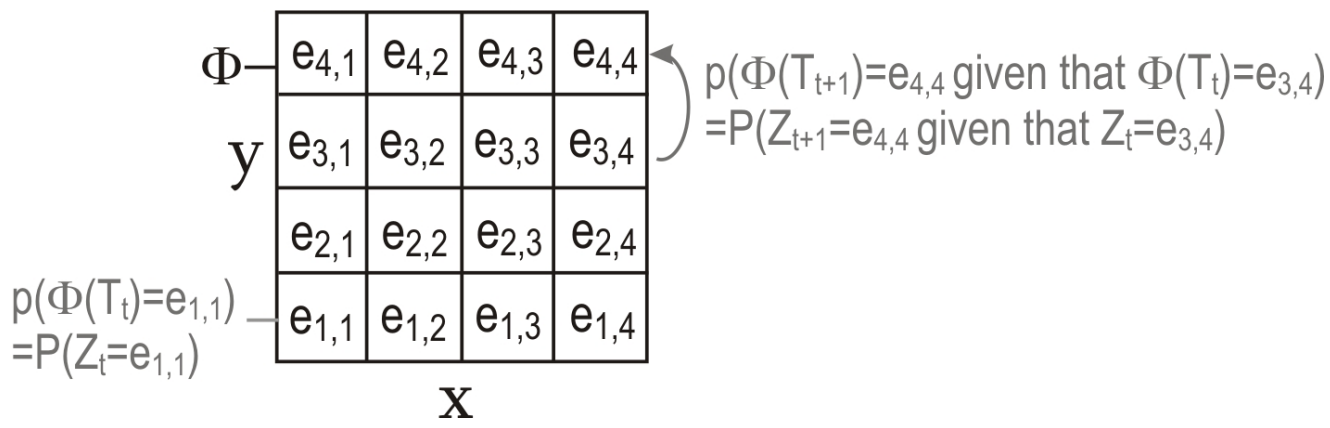

Figure 4: Observing the baker's system leads to the stochastic model $\left\{\Phi\left(T_{t}\right)\right\}$

this observation level, the physical system of the particle bouncing on mirrors can be described by the deterministic baker's model or by the stochastic model $\left\{\Phi_{16}\left(T_{t}\right)\right\}$.

It is the case that $\left\{\Phi_{16}\left(T_{t}\right)\right\}$ is the Markov model $\left\{W_{t}\right\}$ (for the definition of $\left\{W_{t}\right\}$ see Example 3). Thus the baker's model, relative to $\Phi_{16}$, and the Markov model $\left\{W_{t}\right\}$, relative to the identity function, are observationally equivalent. Now, there can be an explanation of why the time series of Figure 1 can arise from both the baker's model, relative to $\Phi_{16}$, and the Markov model $\left\{W_{t}\right\}$. Figure 1 shows the first 11 iterates of the baker's map starting in $(0.824,0.4125)$ coarse-grained by $\Phi_{16}$, viz. $X=\left(\left(\frac{7}{8}, \frac{3}{8}\right),\left(\frac{5}{8}, \frac{5}{8}\right),\left(\frac{3}{8}, \frac{7}{8}\right),\left(\frac{5}{8}, \frac{3}{8}\right),\left(\frac{1}{8}, \frac{5}{8}\right),\left(\frac{3}{8}, \frac{3}{8}\right),\left(\frac{5}{8}, \frac{1}{8}\right),\left(\frac{3}{8}, \frac{5}{8}\right)\right.$, $\left.\left(\frac{7}{8}, \frac{3}{8}\right),\left(\frac{7}{8}, \frac{5}{8}\right),\left(\frac{7}{8}, \frac{7}{8}\right)\right)$. But $X=\left(e_{2,4}, e_{3,3}, e_{4,2}, e_{2,3}, e_{3,1}, e_{2,2}, e_{1,3}, e_{3,2}, e_{2,4}, e_{3,4}, e_{4,4}\right)$, which are the first eleven outcomes of a realisation of the Markov model $\left\{W_{t}\right\}$.

Clearly, the Markov model $\left\{W_{t}\right\}=\left\{\Phi_{16}\left(T_{t}\right)\right\}$ is nontrivial (where a stochastic model is nontrivial if it is not trivial; for a trivial stochastic model the transition probabilities, i.e., the probabilities that any arbitrary value follows another arbitrary value, are trivial ( 0 or 1$)$ ). This indicates observational equivalence to a nontrivial stochastic model. This raises the question whether there are general results that, given a deterministic model, there are observationally equivalent nontrivial stochastic models $\left\{\Psi\left(T_{t}\right)\right\}$. This is an important question because if $\left\{\Psi\left(T_{t}\right)\right\}$ is trivial, then it could be argued that $\left\{\Psi\left(T_{t}\right)\right\}$ is really a deterministic model (though formally it is a stochastic model), and that there is no observational equivalence between deterministic and indeterministic models. Yet several results show that $\left\{\Psi\left(T_{t}\right)\right\}$ is often nontrivial. What follows is a result that is relevant in the rest of the paper (cf. Werndl 2009a, 2011).

Theorem 1 If for the measure-preserving deterministic model $\left(M, T_{t}, p\right)$ there does not exist an $n \in \mathbb{N}$ and $a C \subseteq M, 0<p(C)<1$, such that $T_{n}(C)=C$, then

quently, scientists do not test the deterministic and stochastic models describing these systems by deriving probabilistic predictions from them. However, the example of the particle bouncing on mirrors is easy to understand and billiards with convex obstacles are the prime systems discussed by the extant philosophical literature. Thus, instead of referring to other examples, this paper simply assumes that scientists test in this way. 
for any arbitrary nontrivial finite-valued observation function $\Psi$ the stochastic model $\left\{Z_{t}\right\}=\left\{\Psi\left(T_{t}\right)\right\}$ is nontrivial in the following sense: For every $k \in \mathbb{N}$ and $t \in \mathbb{Z}$ there are $e_{i}, e_{j} \in E$ such that $0<P\left(Z_{t+k}=e_{i}\right.$ given that $\left.Z_{t}=e_{j}\right)<1$.

In practice, observations are finite-valued and therefore Theorem 1 says that every observation in practice leads to a nontrivial stochastic model. Theorem 1 applies to the baker's model (Example 1) and to Newtonian models of billiards with convex obstacles (Example 2) (Ornstein and Galavotti, 1974). Thus, regardless which finite-valued observation function is applied to the baker's model or a billiard model, nontrivial stochastic models are obtained. Theorem 1 also applies to several other deterministic models used in science. For example, to hard-ball models important in statistical mechanics, in particular, to two hard balls moving in a box and the motion of $N$ hard balls moving on a torus for almost all values $\left(m_{1}, \ldots, m_{N}, r\right)$, where $m_{i}$ is the mass of the $i$-th ball and $r$ is the radius of the balls, $N \geq 2$ arbitrary (Simányi 1999, 2003); to many other types of billiard models (Chernov and Markarian 2006); and also to dissipative models such as the Hénon model and the Lorenz model which have been used to model weather dynamics and waterwheels (Benedicks and Young 1993, Lorenz 1963, Luzzatto et al. 2005). Furthermore, because of the extreme mathematical difficulties, for many models it is conjectured but not proven that Theorem 1 applies. For example, it is conjectured that Theorem 1 applies to any number of hard balls (greater than 3) in a box and the motion of KAM-type models restricted to regions of unstable behaviour (Berkovitz et al. 2006, 679-680).

The results presented to this point are about how one can find observationally equivalent stochastic models, when starting with deterministic models. There are also results about the converse question of how, when starting with stochastic models, one can find observationally equivalent deterministic models. The underlying construction is the same. Namely, given a stochastic model $\left\{Z_{t}\right\}$ and an observation function $\Gamma$, one finds a deterministic model $\left(M, T_{t}, p\right)$ and an observation function $\Phi$ such that $\left\{\Gamma\left(Z_{t}\right)\right\}=\left\{\Phi\left(T_{t}\right)\right\}$. Because the specific results about this converse question are not of significant importance for what follows, this section only briefly mention two results. First, given any stochastic model and any observation function $\Gamma$, it is possible to construct a deterministic model - called the deterministic representation - and a specific observation function $\Phi_{0}$ such that the deterministic representation relative to $\Phi_{0}$ is observationally equivalent to the stochastic model relative to $\Gamma$. However, the phase space of the deterministic representation is the set of all possible realisations of the stochastic model, and thus the deterministic representation involves a cheat and is not used in science (cf. Werndl 2009a, 2011). The other extant results show that specific deterministic models are observationally equivalent to certain kinds of stochastic models. For instance, several deterministic models used in science are observationally equivalent to Markov models (Example 3). As explained above, the baker's model yields a Markov model relative to $\Phi_{16}$. 
Similarly, billiards with convex obstacles (Example 2) yield Markov models relative to specific observation functions (cf. Ornstein and Weiss 1991, Werndl 2009a, 2011).

\section{Choice and Underdetermination between De- terministic and Indeterministic Models}

As discussed, deterministic and stochastic models are often observationally equivalent. More specifically, a deterministic model $\left(M, T_{t}, p\right)$ relative to $\Phi$ and a stochastic model $\left\{\Psi\left(T_{t}\right)\right\}$ relative to $\Gamma$ are often observationally equivalent. Observational equivalence in this sense holds relative to the observation level determined by the observation functions $\Phi$ and $\Gamma$ and relative to the predictions which are derivable from the deterministic and the stochastic model. Therefore, if $\Psi$ corresponds to an observation finer than currently possible (or actual) observations, then the deterministic and the stochastic model will be compatible with all currently possible (or actual) observations of the physical system. For example, if $\Phi_{16}$ corresponds to an observation at least as fine as the currently possible observations, then the baker's model (Example 1) and the Markov model $\left\{\Phi_{16}\left(T_{t}\right)\right\}$ (see Example 3) are compatible with the currently possible observations of the particle bouncing on mirrors (Figure 1 shows an example).

If there is observational equivalence, there is a choice between models. The question then arises: Is the deterministic model or the stochastic model preferable relative to evidence? Underdetermination arises if data equally supports the deterministic model and the stochastic model. Underdetermination is between models and not - as in usual discussions - between theories (cf. Stanford 2001). Theories provide a general scheme which can be used to obtain models of many different physical systems; e.g., from Newtonian theory are obtained models about billiard systems, planetary systems or systems of air currents (dependent on the exact force term, etc.). A model is only about one system, e.g., about a ball moving on a table with convex obstacles. ${ }^{4}$ Back to our example, there is a choice between the baker's model and the Markov model $\left\{\Phi_{16}\left(T_{t}\right)\right\}$. Which model, then, is preferable relative to evidence? If both are equally supported by evidence, there is underdetermination. For this and similar cases, scientists face the question of which model to use to describe a physical system. Scientists should, and want to, choose the model which is best supported by evidence. Hence, it is decisive which model is preferable relative to evidence. Also, in some situations, scientists have a clear preference and choose one of

\footnotetext{
${ }^{4} \mathrm{~A}$ physical system is the correspondence in reality to a model. Hence, a physical system instantiates not only one process but many different processes (namely, all processes arising from different initial conditions).
} 
the models, e.g., the Newtonian model of billiards rather than any of the corresponding stochastic models. How can their choice be justified? Ideally, their choice should be based on evidence, and again the question arises which model is preferable relative to evidence.

It is important to see that observational equivalence, and compatibility with the currently possible (or actual) observations, may imply, but does not necessarily imply, underdetermination. There are two reasons why there might be no underdetermination: (i) observations at a lever finer than $\Psi$ can constitute evidence only for the deterministic or stochastic model; (ii) second, predictions which are not derivable from the deterministic or stochastic model can constitute evidence only for the deterministic or the stochastic model. That these two reasons can make one of the models preferable is clearly explained in this and the next section.

To properly discuss the central question, there needs to be a differentiation between different kinds of choice and underdetermination. To avoid any potential confusion, this paper distinguishes between models at different levels of reality versus models at the same level of reality. First, for models at different levels of reality the deterministic model describes the evolution of states at the lower level, and the stochastic model describes the evolution of states at the higher level. Furthermore, the state at the higher level is a function of the state at the lower level; hence, the states at the higher and the states at the lower level are related by an observation function $\Psi$. A paradigm example is Boltzmannian statistical mechanics. Here the states at the lower level are represented by the position and velocity coordinates of the particles of a gas, and the states at the higher level are represented by values of variables such as temperature, pressure and volume. The model at the lower level is deterministic while the one at the higher level is stochastic (cf. Frigg 2008, Section 3.2). Second, the deterministic and the stochastic model can be about the same level of reality; e.g., they both describe the evolution of the position and the velocity of a billiard ball. The previous philosophical discussion is about the second kind of choice (Hoefer 2008, Suppes 1993, Suppes and de Barros 1996, Winnie 1998). Hence, what follows will be about this second kind of choice.

Whether a deterministic or a stochastic model is preferable relative to evidence depends on the class of observations considered. ${ }^{5}$ There are four main cases: First, there is choice relative to currently possible observations, i.e., currently possible observations given the available technology. If the evidence

\footnotetext{
${ }^{5}$ The literature on underdetermination also highlights that whether there is underdetermination can depend on the auxiliary hypotheses which are adopted when testing theories, such as hypotheses about measurement apparatus (Ladyman 2002, Section 6.4.1, Laudan and Leplin 1991). The assumption of this paper is that observations can be modelled by observation functions. Given this, all auxiliary hypotheses adopted when testing the predictions of the deterministic and the stochastic model seem to have the same effect on the deterministic and stochastic model. This is assumed in the following sections.
} 
equally supports a deterministic and a stochastic model, there is underdetermination relative to currently possible observations (cf. Laudan and Leplin 1991). Second, there is choice relative to the actual observations. Here there can be underdetermination relative to the actual observations (cf. Ladyman 2002, Section 6.1.1). Third, there is choice relative to observations which are possible in principle where there are limits, in principle, on observational accuracy. Fourth, there is choice relative to observations which are possible in principle where there are no limits, in principle, on observational accuracy. (Here, although observation can never be made with infinite precision, observations which are possible in principle allow the researcher to come arbitrarily close to these infinitely precise values.) For the third and fourth case there could be underdetermination relative to observations which are possible in principle. All four cases are discussed. Note that of relevance in practice is the choice relative to currently possible observations (or actual observations) (cf. Laudan and Leplin 1991). In what follows, the case where the deterministic model $\left(M, T_{t}, p\right)$ and all of the stochastic models $\left\{\Psi\left(T_{t}\right)\right\}$ are disconfirmed by the relevant observations is put aside (simply, none of the models is acceptable).

The first significant result is that there is no underdetermination for the fourth case, i.e., relative to observations which are possible in principle and where there are no limits, in principle, on observational accuracy. If it is found that always finer observations of the state can be made, the deterministic model is preferable because only the deterministic model always allows that finer observations can be made. However, suppose that the possible observations show that there are no other states apart from the states corresponding to the values of a specific observation function $\Psi$. Then, the stochastic model $\left\{Z_{t}\right\}=\left\{\Psi\left(T_{t}\right)\right\}$ is preferable because only this model does not have more states and is in agreement with the observations. Here there is no underdetermination for the reason (i) mentioned above: Observations at a lever finer than $\Psi$ constitute evidence only for a deterministic or a stochastic model.

Yet, it is not easy to dismiss the other kinds of underdetermination. Most importantly, consider the choice between a deterministic model $\left(M, T_{t}, p\right)$ and a stochastic model $\left\{Z_{t}\right\}=\left\{\Psi\left(T_{t}\right)\right\}$ relative to the currently possible observations (where $\Psi$ is an arbitrary function). There are two cases: either $\Psi$ is at least as fine as the currently possible observations or not. The conclusions are straightforward for the second case. If the possible observations show that there are no other states apart from the states corresponding to the values of $\Psi$, then $\left\{\Psi\left(T_{t}\right)\right\}$ is preferable because only this stochastic model agrees with the observations (underdetermination fails again for the reason (i), as previously mentioned). However, if the possible observations show that there are more states than the values given by $\Psi$, then $\left\{Z_{t}\right\}=\left\{\Psi\left(T_{t}\right)\right\}$ cannot correctly describe the relevant level of reality, but all that can be concluded is that either a deterministic model or a finer stochastic model correctly describes this 
level of reality. The more interesting situation is when $\Psi$ is at least as fine as the currently possible observations, and in the rest of the paper this is the assumption. Here it will not be possible to find out whether there are more states than the values given by $\Psi$. The predictions of the deterministic model and of the stochastic model $\left\{Z_{t}\right\}=\left\{\Psi\left(T_{t}\right)\right\}$ then agree at all currently possible observation levels. For instance, if $\Psi:=\Phi_{16}$ corresponds to an observation as least as fine as the currently possible observations, then the baker's model and $\left\{\Phi_{16}\left(T_{t}\right)\right\}$ agree at all currently possible observation levels. If these predictions are confirmed and other evidence does not favour a model, there is underdetermination relative to the currently possible observations. (Because $\Psi$ can vary, there can be underdetermination between a deterministic model $\left(M, T_{t}, p\right)$ and all the stochastic models $\left\{\Psi\left(T_{t}\right)\right\}$, where $\Psi$ is an arbitrary fine-enough observation function.) Underdetermination can still be avoided for reason (ii), mentioned above, namely if predictions which are not derivable from the deterministic or stochastic model constitute evidence for only one of the models (the next section discusses such a case).

Similarly for the choice relative to the actual observations and the choice relative to observations which are possible in principle where there are limits, in principle, on observational accuracy (where $\Psi$ is an arbitrary function and $\left.\left\{Z_{t}\right\}=\left\{\Psi\left(T_{t}\right)\right\}\right)$ : Either $\Psi$ is at least as fine as the actual observations or the observations which are possible in principle, or not. The second case is straightforward. If observations show that there are exactly the states corresponding to $\Psi$, then $\left\{Z_{t}\right\}=\left\{\Psi\left(T_{t}\right)\right\}$ is preferable. If the observations indicate that there are more states than values corresponding to $\Psi$, either a deterministic model or a finer stochastic model is correct. Again, the more interesting situation is when $\Psi$ is at least as fine as the actual observations or the observations which are possible in principle, and in the rest of the paper this is assumed. Here the predictions of the deterministic model and of the stochastic model $\left\{Z_{t}\right\}=\left\{\Psi\left(T_{t}\right)\right\}$ agree for all actual observation levels or observation levels which are possible in principle. If other evidence does not favour a model, there is underdetermination relative to the actual observations or the observations which are possible in principle. (Because $\Psi$ can vary, there can be underdetermination between a deterministic model and all the stochastic models $\left\{\Psi\left(T_{t}\right)\right\}$, where $\Psi$ is fine enough.) Again, underdetermination may be avoided for reason (ii) mentioned above.

The next section presents, for an important class of models, a new answer to the central model-preference question. Section 6 then discusses the extant philosophical literature. The paper is organised in this way because the novel answer provides important background for the criticism of the philosophy literature. What should be noted, though, is that the new answer was developed as a response to the extant answers which, in the view of this paper, are unsatisfactory for the types of choice arising in practice. 


\section{A New Argument Against Underdetermina- tion Based on Indirect Evidence}

As explained in the previous section, there is no underdetermination relative to observations which are possible in principle where there are no limits, in principle, on observational accuracy. The new argument applies to choice relative to currently possible observations, relative to actual observations or relative to observations which are possible in principle where there are limits, in principle, on observational accuracy. (The argument for the currently possible observations is outlined, but it carries over to the actual observations and the observations which are possible in principle). The focus is on a case which, as will be seen later, generalises the main example discussed by the extant philosophical literature - the physical system of a billiard ball moving on a table with convex obstacles. Namely, this paper concentrates on the case where the predictions of a Newtonian deterministic model $\left(M, T_{t}, p\right)^{6}$ and of a stochastic model $\left\{Z_{t}\right\}=\left\{\Psi\left(T_{t}\right)\right\}$ which is not based on any theory are confirmed, thereby there is a choice between $\left(M, T_{t}, p\right)$ and $\left\{Z_{t}\right\}$ (here, $\Psi$ is an arbitrary function finer than the currently possible observation functions). I will identify conditions showing that the Newtonian deterministic model is preferable. It might seem intuitive that the deterministic model is preferable, but careful arguments are needed to show whether or not this intuition is correct. As will be shown in Section 6, arguments that the deterministic model is preferable can easily go wrong. The argument will finally be generalised to a much broader class of cases.

Note that $\left(M, T_{t}, p\right)$ and $\left\{Z_{t}\right\}$ give the same predictions at the currently possible observation level. To therefore avoid the conclusion of underdetermination, observations are needed that do not follow from these models and constitute evidence only for the deterministic or the stochastic model. This paper argues that such evidence can be found by appealing to other Newtonian models. Here underdetermination fails for the reason (ii) outlined in Section 4. At this point, it should be emphasised that for the new argument against underdetermination other systems are needed in which the theory is confirmed. Hence, the argument does not apply to the choice between models which describe the whole world.

Focus now turns to the choice between the Newtonian deterministic model and the stochastic model. What does it mean that a model $F$ is based on a theory? ${ }^{7}$ This means that either (i) the model $F$ is derived from the postulates of the theory and the additional specific assumptions about the physical system

\footnotetext{
${ }^{6}$ Here, the assumption is that the Newtonian model can be written as a measure-theoretic deterministic model $\left(M, T_{t}, p\right)$. As explained in Section 2.1, e.g., all Newtonian deterministic models which model energy-conserving systems are of this form.

${ }^{7}$ When a model is based on a theory this means that this is relative to state-of-the-art of scientific theorising.
} 
that go beyond these postulates. For example, deterministic models of billiards with convex obstacles are based on Newtonian theory in this sense. The additional specific assumptions are crucial because the model cannot be derived from the postulates of the theory alone; specific assumptions about the physical system are always needed to arrive at a model. Or (ii) there is a model $C$ describing another level of reality and which is derivable from the postulates of the theory and the additional specific assumptions about the physical system. Furthermore, it is specified how the states of $C$ correspond to the states at the other level of reality. With this correspondence rule, the model $F$ is derivable from $C$. Examples are stochastic models about the evolution of macrostates, represented by values of variables such as local temperature, pressure and volume, in Boltzmannian statistical mechanics. Here for instance, the stochastic model is derived from the Newtonian model of the motion of the particles of a gas and the correspondence rule between the states of particles and macrostates.

The new answer to the central question involves the idea of indirect evidence. This idea can be explained with an example given by Laudan and Leplin (1991, 461-462). The theory of continental drifts states the following:

TC: Every region of the surface of the earth has occupied latitudes as well as longitudes significantly different from those it now occupies.

The following two hypotheses follow from $T C$ :

$\mathrm{H}_{1}$ : Each part of a continent has seen markedly different climates in its history.

$\mathrm{H}_{2}$ : In any region of the earth the current alignment of the earth's magnetic pole of the magnetism of iron-bearing rock is markedly different from the alignment of the magnetic rocks of the region from earlier periods.

In the 1950s and 1960s data $e$ about remnant magnetism accrued which supported $\mathrm{H}_{2}$ and thus also TC. According to Laudan and Leplin, because $e$ supports TC, $e$ provides (and was regarded as providing) evidence for $\mathrm{H}_{1}$, even though $\mathrm{H}_{1}$ does not entail $e$. $e$ is an example of indirect evidence for $\mathrm{H}_{1}$.

There are many other examples of indirect evidence, and indirect evidence is regarded as an important form of evidence (Laudan and Leplin 1991; Moretti 2007; Okasha 1998, Okasha 2002). Importantly, as noted by Laudan and Leplin (1991), indirect evidence can block underdetermination. To come back to the example: suppose there is a hypothesis $\mathrm{H}^{\prime}$ from which the same predictions are derivable than from $\mathrm{H}_{1}$ but which does not follow from $\mathrm{TC}$ or any other more general theory. Then the evidence favours $\mathrm{H}_{1}$ over $\mathrm{H}^{\prime}$ because there is indirect evidence only for $\mathrm{H}_{1}$ but not for $\mathrm{H}^{\prime}$; hence, there is no underdetermination.

One main claim made by this paper is that indirect evidence can also block underdetermination for the choice between models. This can be illustrated with the example of a Newtonian model of a billiard with convex obstacles 
(Example 2). Here the predictions of the Newtonian model $\left(M, T_{t}, p\right)$ and of the stochastic model $\left\{\Psi\left(T_{t}\right)\right\}$ are confirmed by the observations. Now consider other Newtonian models which invoke similar additional assumptions about the physical systems such as Newtonian models of billiards without obstacles, Newtonian models of billiards on a table of another shape and Newtonian models of billiards with several balls. ${ }^{8}$ Suppose that the predictions of these other models are confirmed. Because Newtonian theory and the similar additional assumptions about the physical systems are well confirmed, the evidence for these other models provides indirect evidence for the deterministic model of billiards with convex obstacles. There is no indirect evidence for the stochastic model $\left\{\Psi\left(T_{t}\right)\right\}$. Therefore, the deterministic model is preferable. Likewise, for the baker's model of a particle bouncing on several mirrors, consider other Newtonian models which invoke similar additional assumptions such as Newtonian models of a particle bouncing on different kinds of mirrors, or Newtonian models of several particles bouncing on mirrors. If confirmed by observation, the observations provide indirect evidence for the baker's model, making the baker's model preferable.

The general argument about the choice between the Newtonian deterministic model $\left(M, T_{t}, p\right)$ and the stochastic model $\left\{\Psi\left(T_{t}\right)\right\}$ is as follows. By assumption, the predictions of the Newtonian model and the stochastic model are confirmed by the observations. There will then usually be other Newtonian deterministic models which invoke similar additional assumptions about the physical system as $\left(M, T_{t}, p\right)$, and which are also confirmed by the observations. In these cases, because both Newtonian theory and the additional similar assumptions are well confirmed, the evidence for these other models provides indirect evidence for $\left(M, T_{t}, p\right)$. The stochastic model is not supported by indirect evidence. Consequently, there is more evidence for the deterministic model because it receives indirect evidence from other Newtonian models. Thus the deterministic model is preferable, and there is no underdetermination. ${ }^{9}$ As previously mentioned, underdetermination fails here for reason (ii) outlined in Section 4: Observations from other models constitute evidence only for the deterministic model.

What does indirect evidence amount to in these cases? Note that two models are derivable from the same statement cannot be sufficient for the transmission of confirmation from one to the other because this would lead to Hempel's paradox (the absurdity that any two statements confirm each other - Okasha

\footnotetext{
${ }^{8}$ The behaviour of some of these models is very different from that of billiards with convex obstacles; e.g., for a ball moving on a rectangular table without obstacles the motion is integrable and thus not chaotic.

${ }^{9}$ Without question, Newtonian theory has been found to give wrong predictions in certain domains and ranges, e.g., about the precession of Mercury's perihelion. However, Newtonian theory delivers numerous correct predictions in (and is applied in) certain ranges and various domains. There is, therefore, a large amount of evidence supporting Newtonian theory.
} 
1998). ${ }^{10}$ What goes on in these examples can be characterised as follows: There is indirect evidence when the models (predictions all confirmed) are unified by a well confirmed theory and by confirmed similar additional assumptions about physical systems. Here both the well-confirmed theory and the confirmed similar additional assumptions about the physical systems make a contribution to the evidence. So indirect evidence results from the unification by a well confirmed theory and confirmed similar additional assumptions. Thus, this account does not suffer from Hempel's paradox.

It is crucial in this instance that the unifying theory of Newtonian mechanics is well confirmed. ${ }^{11}$ How is Newtonian theory confirmed? Newtonian theory is confirmed by all the models which are derived with the help of Newtonian theory and whose predictions are confirmed. For instance, Newtonian models of billiards with convex obstacles are derived with help of Newtonian theory; hence, the observations which confirm their predictions also confirm Newtonian theory. (Likewise, Laudan and Leplin (1991) argue that the observations about remnant magnetism confirm the theory of continental drift (TC) because the predictions about magnetism are derived from the theory of continental drift). Moreover, Newtonian theory is also confirmed by predictions about symmetries, e.g., that the motion of a billiard ball on a ship which moves with uniform speed on a lake is the same as the motion of a billiard ball on land. Here there are no corresponding predictions on the stochastic side because there is no stochastic theory that can provide predictions about more than one stochastic model.

Given this account of indirect evidence, the presented new argument can be generalised: suppose that one model is based on a well confirmed theory and suppose that there are other models which are based on this theory and which, because they are unified by the theory and by confirmed similar additional assumptions, provide indirect evidence for this model. Further, suppose that the other model is not supported by indirect evidence. Then the model that is supported by indirect evidence is preferable. It should be briefly mentioned that a similar argument can also be made in the case where neither of the two models is theory-based but only one model receives indirect evidence from models invoking similar additional assumptions. Namely, suppose that one model is supported by indirect evidence from models which invoke confirmed similar additional assumptions about the physical systems and that the other model is not supported by indirect evidence. Then the model that is supported by indirect evidence is preferable. ${ }^{12}$ It is important to realise that in these generalisations

\footnotetext{
${ }^{10}$ Statement $A$ confirms itself; $A$ derives from $A \wedge B$ where $B$ is any statement; $B$ derives from $A \wedge B$. Consequently, $A$ confirms $B$.

${ }^{11}$ Models can easily be unified by theories which simply accommodate predictions of the models (e.g., by saying that a creator decided that all these models will become true). In these cases there cannot be indirect evidence. Hence, what is needed is a well confirmed theory such as Newtonian theory and not a theory which only accommodates the predictions.

${ }^{12}$ For instance, such an argument could be used to substantiate the claim that the de-
} 
indirect evidence might well lead to the preference for the stochastic model.

In particular, the specific case of the choice between Newtonian deterministic models and stochastic models which are not supported by indirect evidence was discussed because it generalises the examples of the extant philosophy literature. For this specific case Newtonian theory leads to the preference for deterministic models. However, in other cases Newtonian theory can lead to the preference for stochastic models. Let me give two examples. For instance, consider the choice between a stochastic statistical mechanical model of the evolution of the macrostates of a gas (this model is based on Newtonian theory ${ }^{13}$ ) and the deterministic representation of this stochastic model. Then, at the level of reality of the macrostates, the stochastic model can be preferable because of indirect evidence. Second, consider a stochastic model where the motion of a particle is described by Newtonian theory with a stochastic force term (an example is a model arising from the Langevin equation). Given the choice between this stochastic model and its deterministic representation, indirect evidence can lead to the preference for the stochastic model.

This new argument against underdetermination appeals to indirect evidence, and indirect evidence has already been used in the literature to argue against undetermination (Laudan and Leplin 1991; Okasha 2002; Stanford 2001). However, the differences are as follows: First, indirect evidence has never been considered in the debate about the choice between deterministic and indeterministic models. Second, this argument is about choice between models and not about the choice between theories. As explained, theories provide a scheme which can be used to obtain models of many physical systems, while a model is only about one system.

Now that the new answer has been presented, this paper turns to the extant philosophical literature. Two main papers concerning the initial central question, namely Winnie (1998) and Hoefer (2008), are discussed.

\section{Discussion of the Previous Philosophical Lit- erature}

\subsection{Winnie's Argument for the Deterministic Model}

Winnie (1998) aims to provide sufficient conditions under which the deterministic model is preferable. His argument is applied to choice between Newtonian deterministic models $\left(M, T_{t}, p\right)$ and stochastic models obtained by applying a

terministic model of population dynamics presented by May (1976) is preferable over the corresponding stochastic models.

${ }^{13}$ The stochastic model is based on Newtonian theory in the second sense, i.e., by involving a correspondence rule between microstates and macrostates. 
finite-valued observation function $\Psi$ to $\left(M, T_{t}, p\right)$. His main example is the physical system of a billiard with convex obstacles. His argument is as follows: Suppose one is given a deterministic model $\left(M, T_{t}, p\right)$ and a nontrivial stochastic model $\left\{Z_{t}\right\}=\left\{\Psi\left(T_{t}\right)\right\}$, where $\Psi$ is an arbitrary finite-valued observation function. ${ }^{14}\left\{Z_{t}\right\}$ is obtained by applying $\Psi$ to $\left(M, T_{t}, p\right)$. This means that the deterministic model is more informative because it also provides all information at a finer level than $\Psi$, e.g., about the predictions obtained when observation functions finer than $\Psi$ are applied. Thus the deterministic and stochastic models do not give the same predictions and it can be determined when the deterministic model is preferable and when there is no underdetermination. The following quote illustrates that Winnie endorses what can be considered as the 'information argument':

Some deterministic systems, when partitioned, generate stochastic processes. No one of these stochastic processes can, however, generate the deterministic flow. The deterministic flow is, if you like, a recipe for generating stochastic processes, none of which can, in return, generate its parent flow. [...] The deterministic model thus outstrips any single Markov model in its conceptual and predictive power. (Winnie 1998, 317)

Winnie is correct to claim that the deterministic model is more informative. There is an asymmetry because the stochastic model is generated from the deterministic model (in the sense that $\left\{Z_{t}\right\}=\left\{\Psi\left(T_{t}\right)\right\}$ arises by applying the observation function $\Psi$ to $\left.\left(M, T_{t}, p\right)\right)$ and not the other way round. Winnie (1998) seems to be concerned with observations which are possible in principle where there are no limits, in principle, on observational accuracy. For this case, the information argument succeeds in identifying conditions under which the deterministic model is preferable. The observations which are possible in principle confirm all of the structure provided by the deterministic model about levels finer than $\Psi$ in case finer observations can always be made. And, as argued in Section 4 , if finer observations of the states can always be made, then the deterministic model is preferable because only the deterministic models allows that finer observations can always be made. ${ }^{15}$ For example, if billiards with convex obstacles are deterministic, only the Newtonian deterministic models agree with all observations which are possible in principle and, thus, are preferable.

Winnie's argumentation is successful. Yet the question arises whether the information argument also works for the other cases. Does it work for the cases

\footnotetext{
${ }^{14}$ Winnie only considers finite-valued observation functions $\Psi$, but all the claims in this section also hold for any function $\Psi$ such that $\left\{Z_{t}\right\}=\left\{\Psi\left(T_{t}\right)\right\}$ is nontrivial.

${ }^{15}$ So Winnie has already argued what was highlighted in Section 4: Suppose the concern is in observations which are possible in principle where there are no limits, in principle, on observational accuracy. Then, if the phenomenon is deterministic, the possible observations show that the deterministic model is preferable.
} 
of concern in practice, viz. the choice relative to currently possible observations or actual observations, and what about the choice relative to observations which are possible in principle where there are limits, in principle, on observational accuracy? The rest of this section argues that the information argument is not successful in these cases. This is important because Winnie develops his argument as a criticism of Suppes (1993) and Suppes and de Barros (1996). These papers defend the claim that there is underdetermination. Suppes (1993) and Suppes and de Barros (1996) do not state explicitly what kind of choice they are concerned with, but they repeatedly highlight that there is a choice between deterministic and stochastic models only because of limitations in observational accuracy. It therefore seems plausible that Suppes and de Barros were concerned with currently possible observations (or similar). If so, then Winnie's criticism misses the target because it fails for the kind of choice Suppes and de Barros were concerned with. Note that, as argued in Section 4, in certain cases there is no underdetermination between deterministic and stochastic models relative to the currently possible observations. Thus, Suppes and de Barros' claim that there is underdetermination relative to the currently possible observations cannot be generally correct.

Following on from this assessment, the focus is on choice relative to currently possible observations (but the argument carries over to choice relative to actual observations and to choice relative to observations which are possible in principle where there are limits, in principle, on observational accuracy). By assumption, $\Psi$ is an arbitrary finite-valued observation function where the currently possible observations do not indicate whether there are more states than the ones given by $\Psi$. Thus the predictions of $\left(M, T_{t}, p\right)$ and of $\left\{Z_{t}\right\}=\left\{\Psi\left(T_{t}\right)\right\}$ agree at all currently possible observation levels. Here the information argument is that the deterministic model is still preferable because it provides more information. First, it should be noted that these reasons based on 'providing more information' are non-evidential (because the structure of the deterministic model finer than $\Psi$ is not empirically accessible). The central question is which model is preferable relative to evidence and this argument does not answer the question.

There is also another worry: While it is true that the deterministic model provides more information, this information might not be of any value. To stress the point, suppose that the stochastic model $\left\{Z_{t}\right\}=\left\{\Psi\left(T_{t}\right)\right\}$, where $\Psi$ is a specific finite-valued observation function, is the correct model (this can be the case - the predictions derived from the deterministic model and the stochastic model agree at all currently possible observation levels). The deterministic model still provides more information, yet this is not of any value because it does not correspond to anything in the world, and so should not be taken as a reason to prefer the deterministic model. Similarly, the deterministic representation (see Section 3) provides more information. Yet, intuitively, this 
does not make it preferable over the stochastic model because it is questionable whether this additional information is of any value.

To further substantiate the claim that the information argument is untenable for choice relative to currently possible observations, here is an example where the premises of this argument are true but the conclusion is not. This example involves indirect evidence as discussed in Section 5. Suppose that the predictions of the deterministic model $\left(M, T_{t}, p\right)$ and of the stochastic model $\left\{Z_{t}\right\}=\left\{\Psi\left(T_{t}\right)\right\}$ are confirmed (where $\Psi$ is a specific observation function). Clearly, $\left(M, T_{t}, p\right)$ contains more information and the premises of the information argument are true. Now suppose that $\left\{Z_{t}\right\}$ is based on a well confirmed theory $G$, and that there are similar models based on $G$ which are also well confirmed and which provide indirect evidence for $\left\{Z_{t}\right\}$ (there is indirect evidence because these models are unified by the well confirmed theory $G$ and invoke confirmed similar additional assumptions about the physical systems). Further, suppose that there is no indirect evidence for $\left(M, T_{t}, p\right)$. Then the stochastic model $\left\{Z_{t}\right\}$ is preferable relative to evidence, and the conclusion of the information argument is not true.

In summary, Winnie's argument succeeds for choice relative to in observations which are possible in principle (the case was concerned with), but it does not work for the kinds of choice arising in practice.

\subsection{Hoefer's Argument for the Deterministic Model}

Hoefer (1998) presents an argument for the deterministic model, with the intention to show that the Newtonian deterministic model $\left(M, T_{t}, p\right)$ is preferable over any of the stochastic models obtained by applying an observation function $\Psi$ to $\left(M, T_{t}, p\right)$. (His main example is also the physical system of a billiard with convex obstacles.) Consider Hoefer's (2008) claim about the choice between Newtonian deterministic models and the corresponding stochastic models ${ }^{16}$ :

It may well be true that there are some deterministic dynamical systems that, when viewed properly, display behavior indistinguishable from that of a genuinely stochastic process. For example, using the billiard table above [a billiard system with convex obstacles], if one divides its surface into quadrants and looks at which quadrant the ball is in at 30-second intervals, the resulting sequence is no doubt highly random. But this does not mean that the same system, when viewed in a different way (perhaps at a higher degree of precision) does not cease to look random and instead betrays its deterministic nature. [Hoefer 2008, original emphasis]

\footnotetext{
${ }^{16}$ Hoefer's (2008) aim seems to be to summarise Winnie's (1998) argument discussed above. However, the argument Hoefer makes is quite different from Winnie's argument.
} 
What this quote expresses depends on the meaning of the word 'random', which is understood very differently in the literature. Hoefer (2008) in the paragraphs preceding this quote uses the word 'random' synonymously to 'stochastic'. The interpretation of 'random' as 'stochastic' is confirmed by the first sentence of the above quote, where Hoefer speaks about a 'genuinely stochastic process'. It can be assumed that 'random' means 'stochastic'. ${ }^{17}$

Then the argument Hoefer makes is as follows: (P1) once a deterministic phenomenon is viewed in the right way, trivial transition probabilities can be obtained; (P2) obtaining trivial transition probabilities indicates that the phenomenon is deterministic. (C) Therefore, the deterministic model is preferable.

Hoefer is silent about the kind of underdetermination he is concerned with. Yet for any of the possible understandings of underdetermination discussed in Section 4, this argument is misguided for two reasons. First, for every deterministic model of billiards with convex obstacles every finite-valued observation function yields a nontrivial stochastic model (cf. Theorem 1 and the discussion thereafter). The possible observations are finite-valued. Thus, there will never be trivial transition probabilities (Premise (P1) is false), and observations of billiards will never cease to look stochastic.

Second, assume that by observing a phenomenon trivial transition probabilities are obtained. This does not imply that the observations derive from a deterministic system, i.e., Premise (P2) is false. Trivial transition probabilities can result from observing a (nontrivial) stochastic system or a deterministic system. Two examples are given to illustrate this: first, a deterministic model which yields trivial transition probabilities; second, a stochastic model which yields trivial transition probabilities.

First, consider a deterministic model $\left(M, T_{t}, p\right)$ describing the motion of a billiard ball on a table where there is a barrier in the middle separating the lefthand side of the table from the right-hand side. If the billiard ball is on one side, it always was and will stay there. Suppose the observation function $\Phi$ tell one whether the ball is on the right- $\left(o_{r}\right)$ or left-hand side $\left(o_{l}\right)$ of the table. Trivial transition probabilitiesare then observed: $p\left(\Phi\left(T_{t+k}\right)=o_{i}\right.$ given $\left.\Phi\left(T_{t}\right)=o_{j}\right)=1$ if $i=j=l$ or $i=j=r$ and $p\left(\Phi\left(T_{t+k}\right)=o_{i}\right.$ given $\left.\Phi\left(T_{t}\right)=o_{j}\right)=0$ otherwise (for all $t, k$ ).

\footnotetext{
${ }^{17}$ From what Hoefer (2008) states, this is by far the most plausible interpretation and has also been suggested to this researcher as the most plausible interpretation by philosophy colleagues. One could also interpret 'random' in Hoefer's quote as meaning that there are probability distributions characteristic of stochastic systems. His argument would then be as follows: Once the observations of a deterministic system are fine enough, there will be observations of probability distributions characteristic of deterministic systems. These specific probability distributions provide evidence that the system is deterministic. Therefore, the deterministic model is preferable. Due to a lack of space, this argument will not be discussed here. The general discussion, though, of Werndl (2011) is easily applied to this argument, showing that this argument does not work.
} 
Second, consider the stochastic model $\left\{Z_{t}\right\}, t \in \mathbb{Z}$, defined as follows (a Markov model - see Example 3): the outcome space is $E=\left\{s_{1}, s_{2}, s_{3}, s_{4}\right\}$, $P\left\{Z_{t}=s_{i}\right\}=1 / 4$ for all $i, 1 \leq i \leq 4$ and all $t ; P\left\{Z_{t}=s_{i}\right.$ given $Z_{t-1}=$ $\left.s_{j}\right\}=1 / 2$ for all $i, j \in\{1,2\}$ and all $t$; and $P\left\{Z_{t}=s_{i}\right.$ given $\left.Z_{t-1}=s_{j}\right\}=1 / 2$ for all $i, j \in\{3,4\}$ and all $t$. This means that $s_{1}$ and $s_{2}$ can be reached from each other but not from $s_{3}$ or $s_{4}$; likewise, $s_{3}$ and $s_{4}$ can be reached from each other but not from $s_{1}$ or $s_{2}$. Thus the stochastic model can be split into two parts: the dynamics involving $s_{1}$ and $s_{2}$ and the dynamics involving $s_{3}$ and $s_{4}$. Now, assume to the stochastic model is applied the observation function $\Gamma:\left\{s_{1}, s_{2}, s_{3}, s_{4}\right\} \rightarrow\left\{o_{1}, o_{2}\right\}, \Gamma\left(s_{1}\right)=\Gamma\left(s_{2}\right)=o_{1}$ and $\Gamma\left(s_{3}\right)=\Gamma\left(s_{4}\right)=o_{2} . \Gamma$ tells one whether the outcome is in $\left\{s_{1}, s_{2}\right\}$ or in $\left\{s_{3}, s_{4}\right\}$. Then one observes trivial transition probabilities: $P\left(\Gamma\left(Z_{t+k}\right)=o_{i}\right.$ given $\left.\Gamma\left(Z_{t}\right)=o_{j}\right)=1$ if $i=j=1$ or $i=j=2$ and $P\left(\Gamma\left(Z_{t+k}\right)=o_{i}\right.$ given $\left.\Gamma\left(Z_{t}\right)=o_{j}\right)=0$ otherwise (for all $t, k)$. However, these trivial transition probabilities are obtained from observing the nontrivial stochastic model $\left\{Z_{t}\right\}$ with $\Gamma$. In conclusion, Hoefer's (2008) argument that the deterministic model is preferable does not work.

\section{Conclusion}

At the outset of this paper, there was a discussion of a time series of observations of the position of a particle (see Figure 1). Scientists aim to find a model which reproduces these observations and yields correct predictions. One of the questions which arises is whether the physical system is better described by a deterministic or an indeterministic model. Intuitively, one might think that the observations only allow for a deterministic or an indeterministic model. However, as has been shown in Section 3, this is not so. In several cases deterministic models and stochastic models are observationally equivalent. Therefore, the central question of this paper concerns which model should be preferred relative to all evidence. If the evidence equally supports both models, there is underdetermination.

Section 4 distinguished between the different kinds of choice and underdetermination these results could pose. Section 5 presented a new answer to the central question: Consider a Newtonian deterministic model $D$. Suppose that there are other Newtonian models $W$ which are also confirmed by observations, and which provide indirect evidence for $D$ (because $W$ and $D$ are unified by the well-confirmed Newtonian theory and invoke confirmed similar additional assumptions about the physical systems). Further suppose that the stochastic model is not supported by indirect evidence. Then the Newtonian model $D$ is preferable. For instance, this argument shows that the Newtonian model of billiards with convex obstacles is preferable to the corresponding stochastic models. The new argument was then generalised: suppose that one of the models is based on a well-confirmed theory, and that there are other models based 
on this theory which invoke confirmed similar additional assumptions and provide indirect evidence for this model. Further, suppose that the other model is not supported by indirect evidence. Then the model supported by indirect evidence is preferable.

Following on from the presentation of the novel answer, Section 6 discussed the previous philosophical literature. Winnie (1998) argues that because the deterministic model is more informative than the stochastic model, one can find out when a system is deterministic, and then the deterministic model is preferable. I argued that Winnie's argument succeeds relative to observations which are possible in principle where there are no limits, in principle, on observational accuracy (the kind of choice Winnie was concerned with). However, for the other kinds of choice, in particular the ones arising in practice, the argument fails. This is so because the argument also applies to situations where the stochastic model is preferable, and the additional information provided by the deterministic model might not be of any value. Hoefer (2008) argues that once one views a deterministic system in the right way, one obtains trivial transition probabilities, indicating that the system is deterministic. However, this argument is misguided because for several deterministic systems there will never be trivial transition probabilities. Furthermore, trivial transition probabilities can also result from observing a nontrivial stochastic system.

The intuition behind Winnie's and Hoefer's arguments is correct - the model based on Newtonian theory is preferable over a non-theory based stochastic model. But these arguments fail to establish the desired conclusion for the kinds of choice of relevance in practice. The proposed new argument, which appeals to indirect evidence, intends to do justice to this intuition and to show why the deterministic model is preferable.

\section{References}

Benedicks, M. and Young, L.-S. (1993) 'Sinai-Bowen-Ruelle-measures for certain Hénon maps'. Inventiones Mathematicae 112, 541-567.

Berkovitz, J., Frigg, R. and Kronz, F. (2006). 'The ergodic hierarchy, randomness and Hamiltonian chaos'. Studies in History and Philosophy of Modern Physics 37, 661-691.

Butterfield, J. (2005). 'Determinism and indeterminism'. Routledge Encyclopaedia of Philosophy Online.

Chernov, N. and Markarian, R. (2006). Chaotic Billiards. American Mathematical Society, Providence. 
Frigg, R. (2008). A field guide to recent work on the foundations of statistical mechanics. In D. Rickles, ed., 'The Ashgate Companion to Contemporary Philosophy of Physics'. Ashgate, London, pp. 99-196.

Hoefer, C. (2008). Causal determinism. In E. Zalta, ed., 'The Stanford Encyclopaedia of Philosophy (Winter 2008 Edition)',

http://plato.stanford.edu/archives/win2008/entries/determinism-causal/, Stanford.

Ladyman, J. (2002). Understanding Philosophy of Science. Routledge, London and New York.

Laudan, L. and Leplin, J. (1991). 'Empirical equivalence and underdetermination'. The Journal of Philosophy 88, 449-472.

Lavis, D. (2011). An objectivist account of probabilities in statistical physics. In C. Beisbart and S. Hartmann, eds, 'Probabilities in Physics', forthcoming. Oxford University Press, Oxford.

Lorenz, E. (1963). 'Deterministic nonperiodic flow'. Journal of the Atmospheric Sciences 20, 130-141.

Luzzatto, S., Melbourne, I. and Paccaut, F. (2005). 'The Lorenz attractor is mixing'. Communications in Mathematical Physics 260, 393-401.

May, R.M. (1976). 'Simple Mathematical Models with Very Complicated Dynamics.' Nature 261, 459-467.

Moretti, L. (2007). 'Ways in which coherence is confirmation conducive'. Synthese 157, 309-319.

Okasha, S. (1998). 'Laudan and Leplin on empirical equivalence'. The British Journal for the Philosophy of Science 48, 251-256.

Okasha, S. (2002). 'Underdetermination, holism and the theory/data distinction'. The Philosophical Quarterly 208, 303-319.

Ornstein, D. and Galavotti, G. (1974). 'Billiards and Bernoulli schemes'. Communications in Mathematical Physics 38, 83-101.

Ornstein, D. and Weiss, B. (1991). 'Statistical properties of chaotic systems'. Bulletin of the American Mathematical Society 24, 11-116.

Petersen, K. (1989). Ergodic Theory. Cambridge University Press, Cambridge.

Simányi, N. (1999). 'Ergodicity of hard spheres in a box'. Ergodic Theory and Dynamical Systems 19, 741-766. 
Simányi, N. (2003) 'Proof of the Boltzmann-Sinai ergodic hypothesis for typical hard disk systems'. Inventiones Mathematicae 154, 123-178.

Stanford, P. (2001). 'Refusing the devil's bargain: What kind of underdetermination should we take seriously?'. Philosophy of Science (Supplement) 68, S1-S12.

Suppes, P. (1993). 'The transcendental character of determinism'. Midwest Studies in Philosophy 18, 242-257.

Suppes, P. and de Barros, A. (1996). Photons, billiards and chaos. In P. Weingartner and G. Schurz, eds, 'Law and Prediction in the Light of Chaos Research'. Springer, Berlin, pp. 190-201.

Werndl, C. (2009a). 'Are deterministic descriptions and indeterministic descriptions observationally equivalent?'. Studies in History and Philosophy of Modern Physics 40, 232-242.

Werndl, C. (2009b). Deterministic versus indeterministic descriptions: Not that different after all? In A. Hieke and H. Leitgeb, eds, 'Reduction, Abstraction, Analysis, Proceedings of the 31st International Ludwig WittgensteinSymposium'. Ontos, Frankfurt, pp. 63-78.

Werndl, C. (2011). 'On the observational equivalence of continuous-time deterministic and indeterministic descriptions'. European Journal for the Philosophy of Science 1 (2), 193-225.

Winnie, J. (1998). Deterministic chaos and the nature of chance. In J. Earman and J. Norton, eds, 'The Cosmos of Science - Essays of Exploration'. Pittsburgh University Press, Pittsburgh, pp. 299-324. 